\title{
Addressing Resident Retention of Musculoskeletal Skills, Knowledge, and Confidence: a Randomized Controlled Study of a Clinic Intervention
}

J Gen Intern Med 36(11):3595-7 DOI: $10.1007 / \mathrm{s} 11606-021-06595-\mathrm{x}$

(c) Society of General Internal Medicine 2021

\section{INTRODUCTION}

Musculoskeletal (MSK) conditions are common reasons for ambulatory visits ${ }^{1}$ yet most internal medicine (IM) residents are uncomfortable and lack proficiency in MSK medicine. ${ }^{2,3}$ Exposure to a MSK rotation produces higher scores on competency examinations but the literature lacks long-term assessments. ${ }^{4,5}$ Our study assessed the 6-month retention of confidence, knowledge, and examination skills after a MSK workshop with participants randomly assigned to a MSK clinic.

\section{METHODS}

We conducted this study at Montefiore Medical Center (Bronx, NY). All second-year IM residents $(n=39)$ participated in a MSK workshop from January through May 2012 and were randomized, with concealed allocation, to subsequently attend a MSK or a non-MSK clinic (Fig. 1). IRB approval was obtained.

The workshop focused on common shoulder and knee conditions and the joint examinations. The residents subsequently attended two half-day weekly sessions for 4 weeks in their specialty clinic. Confidence was assessed using a 5-point Likert scale and board-style written questions assessed knowledge pre- and post-workshop, and 6 months post-intervention. Observed and scored case-based joint examinations were completed at baseline and 6-month post-intervention by one of three authors (CS, AV, DL), blinded to clinic allocation. The outcomes were confidence scores and knowledge post and 6-month post-intervention, and examination skills at 6 months, compared with baseline.

Data were evaluated with the intention to treat (ITT) analysis per randomization. A separate sensitivity analysis (SA) accounted for actual clinic exposure of cross-over residents. Fisher's exact test, $t$ test, and Wilcoxon rank sum test were performed using Stata v15.1.

Received July 29, 2020

Accepted January 1, 2021

Published online January 26, 2021

\section{RESULTS}

All residents completed the immediate post-workshop assessment and 34 (87.2\%) completed the 6-month follow-up (Fig. 1). The median follow-up time was 7 months (IQR 6, 9). Seventy percent attended at least 1 post-workshop clinic session (median $=2.5$; IQR 0, 4.5).

There were no differences at baseline between groups regarding gender, personal injury history, prior MSK training, confidence, knowledge, or examination skills (Table 1). Both groups reported more clinical exposure to knee than shoulder conditions in the prior year. Post-workshop, both groups showed significant improvement in confidence and knowledge scores. At 6 months post-intervention, confidence in both groups returned to baseline with no between-group differences. At 6 months, knee knowledge was retained in both groups and shoulder knowledge in the control group but there were no between-group differences. There was no improvement in the observed shoulder examination in either group. There was improvement in the knee examination only in the intervention group at 6 months and the between-group difference was significant as well $(p=0.02)$. All SA comparisons yielded similar results except that 6 -month post-intervention shoulder knowledge was retained in the exposed but not the non-exposed group $(p=0.03)$. However, there was no between-group difference.

\section{DISCUSSION}

Our 3-h workshop was effective and immediately increased confidence, knowledge, and skill; however, retention in skill required more clinical exposure. The intervention group's 6month retention of knee but not shoulder examination skill may be due to more knee vs. shoulder exposure during our MSK clinics, as occurred in other studies. ${ }^{5}$ Alternatively, these skills may be acquired differently, as other studies have also found post-interventional differences in shoulder compared to knee examination skill. ${ }^{6}$ Retention of 6-month knee knowledge, but not shoulder, in all groups suggests our workshop alone was sufficient instruction for these residents, who all reported more baseline exposure to knee conditions. Our SA showed shoulder knowledge retention only in exposed residents, which may reflect knowledge benefit of the MSK clinic, with the lack of between-group differences likely due to the 


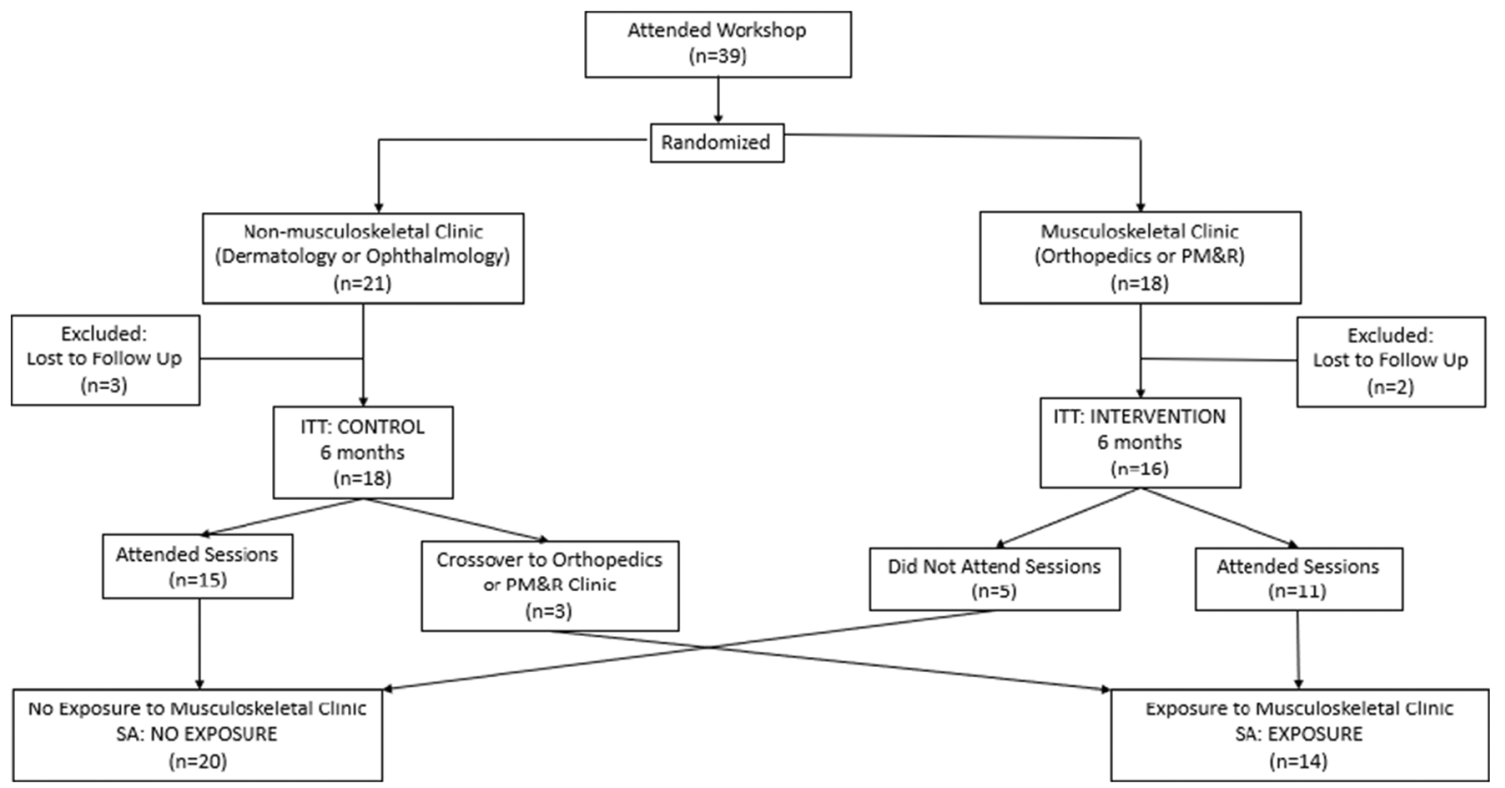

Fig. 1 Flowchart of the study. PM\&R, physical medicine and rehabilitation; ITT intention to treat analysis; SA, sensitivity analysis

Table 1 Results

\begin{tabular}{|c|c|c|c|c|c|c|}
\hline $\begin{array}{l}\text { Control, } n=18 \\
\text { Intervention, } \\
n=16\end{array}$ & $\begin{array}{l}\text { Pre- } \\
\text { intervention }\end{array}$ & $\begin{array}{l}\text { Post- } \\
\text { intervention }\end{array}$ & $\begin{array}{l}\text { Pre vs. } \\
\text { post } \\
p \text { value }\end{array}$ & $\begin{array}{l}\text { 6-month post- } \\
\text { intervention }\end{array}$ & $\begin{array}{l}\text { Pre vs. 6- } \\
\text { month } \\
p \text { value }\end{array}$ & $\begin{array}{l}\text { 6-month post between } \\
\text { group } \\
p \text { value }\end{array}$ \\
\hline \multicolumn{7}{|c|}{ Confidence, mean (Likert scale 1-5) } \\
\hline \multicolumn{7}{|c|}{ Shoulder knowledge } \\
\hline Control $( \pm \mathrm{SD})$ & $2.8(0.9)$ & $3.7(0.8)$ & 0.004 & $2.9(0.8)$ & 0.64 & \multirow[t]{3}{*}{0.92} \\
\hline Intervention & $2.6(0.6)$ & $3.6(0.6)$ & $<0.001$ & $3.0(0.8)$ & 0.11 & \\
\hline \multicolumn{6}{|l|}{$( \pm \mathrm{SD})$} & \\
\hline Shoulder exam & & & & & & \multirow{3}{*}{0.68} \\
\hline Control ( $\pm \mathrm{SD})$ & $2.6(1.0)$ & $3.7(0.8)$ & 0.001 & $2.8(0.8)$ & 0.55 & \\
\hline Intervention & $2.3(0.5)$ & $3.7(0.6)$ & $<0.001$ & $2.8(1.1)$ & 0.17 & \\
\hline \multicolumn{7}{|l|}{$( \pm \mathrm{SD})$} \\
\hline Knee knowledge & & & & & & \\
\hline Control ( $(\mathrm{SD})$ & $3.1(0.7)$ & $3.8(0.7)$ & 0.005 & $3.0(0.8)$ & 0.95 & \multirow{2}{*}{0.23} \\
\hline Intervention & $2.9(0.7)$ & $3.9(0.5)$ & $<0.001$ & $3.4(0.9)$ & 0.13 & \\
\hline \multicolumn{7}{|l|}{$( \pm \mathrm{SD})$} \\
\hline Knee exam & & & & & & \multirow{3}{*}{0.79} \\
\hline Control ( \pm SD) & $2.9(0.7)$ & $3.8(0.7)$ & 0.001 & $3.0(0.8)$ & 0.53 & \\
\hline Intervention & $2.8(0.7)$ & $3.9(0.5)$ & $<0.001$ & $3.1(1.0)$ & 0.39 & \\
\hline \multirow{2}{*}{\multicolumn{7}{|c|}{$\begin{array}{l}( \pm \text { SD) } \\
\text { Knowledge questions median }(\% \text { correct })\end{array}$}} \\
\hline & & & & & & \\
\hline \multicolumn{7}{|c|}{ Shoulder } \\
\hline Control (IQR) & $\begin{array}{l}0.60(0.40, \\
0.70)\end{array}$ & $0.85(0.70,0.90)$ & 0.001 & $0.70(0.60,0.80)$ & 0.02 & \multirow[t]{2}{*}{0.60} \\
\hline $\begin{array}{l}\text { Intervention } \\
\text { (IQR) }\end{array}$ & $\begin{array}{l}0.60(0.50 \\
0.70)\end{array}$ & $0.90(0.75,0.90)$ & 0.001 & $0.70(0.55,0.80)$ & 0.41 & \\
\hline \multicolumn{7}{|l|}{ Knee } \\
\hline Control (IQR) & $\begin{array}{l}0.50(0.38 \\
0.75)\end{array}$ & $0.81(0.63,0.88)$ & 0.003 & $0.75(0.63,0.88)$ & 0.04 & \multirow[t]{2}{*}{0.53} \\
\hline $\begin{array}{l}\text { Intervention } \\
\text { (IQR) }\end{array}$ & $\begin{array}{l}0.50(0.38 \\
0.69)\end{array}$ & $0.81(0.63,0.94)$ & 0.001 & $0.81(0.50,1.00)$ & 0.04 & \\
\hline \multirow{2}{*}{\multicolumn{7}{|c|}{$\begin{array}{l}\text { Observed examination* median } \\
\text { Shoulder }\end{array}$}} \\
\hline Shoulder & & & & & & \multirow{3}{*}{0.81} \\
\hline Control (IQR) & $\begin{array}{l}0.38(0.19 \\
0.50)\end{array}$ & $\mathrm{n} / \mathrm{a}$ & $\mathrm{n} / \mathrm{a}$ & $0.44(0.38,0.63)$ & 0.17 & \\
\hline $\begin{array}{l}\text { Intervention } \\
\text { (IQR) }\end{array}$ & $\begin{array}{l}0.38(0.25 \\
0.53)\end{array}$ & $\mathrm{n} / \mathrm{a}$ & $\mathrm{n} / \mathrm{a}$ & $0.53(0.31,0.63)$ & 0.17 & \\
\hline \multicolumn{7}{|l|}{ Knee } \\
\hline Control (IQR) & $\begin{array}{l}0.39(0.33 \\
0.50)\end{array}$ & $\mathrm{n} / \mathrm{a}$ & $\mathrm{n} / \mathrm{a}$ & $0.47(0.33,0.56)$ & 0.29 & \multirow[t]{2}{*}{0.02} \\
\hline $\begin{array}{l}\text { Intervention } \\
\text { (IQR) }\end{array}$ & $\begin{array}{l}0.44(0.39, \\
0.61)\end{array}$ & $\mathrm{n} / \mathrm{a}$ & $\mathrm{n} / \mathrm{a}$ & $0.61(0.44,0.75)$ & 0.03 & \\
\hline
\end{tabular}

*Observed exam scored out of 1 ( 0 , not completed; 0.5 , completed partially correctly; 1 , completed correctly) 
small sample size. Confidence waned perhaps due to better understanding of the complexity of MSK conditions or it may be that confidence is more associated with an individual's temperament than actual ability.

Strengths of our study include the randomized controlled assignments, concealed allocation, and length of follow-up, which to our knowledge is among the longest period for skill retention assessment. Limitations include the single institution setting, small sample size, possible selection bias regarding residents' motivation in the SA, and variability in the specialty experience.

To achieve long-term retention of knowledge and skill, programs should ensure built-in continual exposure after any short-term intervention. Teaching residents to evaluate shoulder conditions may require more emphasis and/or specific training strategies. Larger studies need to clarify the relationship between knowledge, confidence, and skills.

Cindy Sadikot, $M D^{1}$

Sharon Leung, MD, MS, MHA, FCCP

Athina Vassilakis, $M D, M P H^{3}$

Darlene LeFrancois, $M D^{2}$

${ }^{1}$ Department of Medicine, New York Presbyterian Queens,

New York, NY, USA

${ }^{2}$ Department of Medicine, Montefiore Medical Center, New York, NY, USA

${ }^{3}$ Department of Medicine, Columbia University Medical Center,

New York, NY, USA
Corresponding Author: Cindy Sadikot, MD; Department of Medicine, New York Presbyterian Queens, New York, NY, USA (e-mail: cns9004@nyp.org).

\section{Compliance with Ethical Standards:}

Conflict of Interest: The authors declare that they do not have a conflict of interest.

\section{REFERENCES}

1. Rui P, Okeyode T. National Ambulatory Medical Care Survey: 2016 National Summary Tables. Available from: https://www.cdc.gov/nchs/ data/ahcd/namcs_summary/2016_namcs_web_tables.pdf. Accessed July 25,2020 .

2. Matzkin E, Smith EL, Freccero D, Richardson AB. Adequacy of Education in MSK Medicine. J Bone Joint Surg Am 2005; 87(2):310-4.

3. Houston TK, Connors RL, Cutler N, Nidiry MA. A Primary Care MSK Clinic for Residents: Success and Sustainability. J Gen Intern Med 2004: 19:524-9.

4. Watts SA, Zhang Z . Competency in MSK and Sports Medicine: Evaluating a PGY-1 Curriculum. Fam Med 2011; 43(9):659-63.

5. Wilcox T, Oyler J, Harada C, Utset T. MSK Exam and Joint Injection Technique Training for Internal Medicine Residents. J Gen Intern Med 2006; 21:521-3.

6. Walrod BJ, Schroeder A, Conroy MJ, et al. Does Ultrasound-Enhanced Instruction of MSK Anatomy Improve Physical Examination Skills of FirstYear Medical Students? J Ultrasound Med 2018; 37:225-232

Publisher's Note: Springer Nature remains neutral with regard to jurisdictional claims in published maps and institutional affiliations. 